\title{
Novel risk factors of cardiovascular disease and their associations between obesity, physical activity and physical fitness
}

\author{
Duncan S. Buchan, ${ }^{1}$ Non E. Thomas, ${ }^{2}$ Julien S. Baker ${ }^{1}$ \\ ${ }^{1}$ Health and Exercise Sciences, School of Science, University of the West of Scotland, Hamilton, \\ Scotland; ${ }^{2}$ School of Human Sciences, Swansea University, Swansea, Wales, UK
}

Significance for public health

As the prevalence of traditional CVD risk factors seem to becoming more evident in childhood, it is vital that pre-emptive interventions are implemented early in one's life before the associated cardiovascular complications are established. Childhood is seen as an ideal phase for intervention. This is the stage in one's life where behaviors are established which, if left unabated, may continue into adulthood. Yet, this is also a stage where behaviors can be influenced. Improved understanding of the effects of different physical activity interventions upon CVD risk factors may provide new avenues for intervention that can provide the best means of protection against current and future cardiovascular complications for all youth. Since the role of Physical Education is, to some extent, continually marginalized within the curriculum, it is important that policy makers realise the importance of this setting and its role in the health of school age youth. Thus, further work is necessary within this setting to disentangle the causal relationships between physical activity, physical fitness and CVD risk.

\footnotetext{
Abstract

The prevalence of cardiovascular disease (CVD) is increasing around the globe and is the leading cause of death around the world. Though once thought of as an adult problem, it is now recognised that the early manifestations of disease may occur during childhood. Numerous risk factors have been linked to CVD with much of the research focusing on understanding the prevalence and relationship of traditional risk factors such as dyslipidemia, smoking, diabetes mellitus, hypertension, obesity, psychosocial stress, poor diet, physical inactivity and alcohol consumption to the early etiology of disease. While this line of investigation has greatly enhanced our understanding of the relationship between these risk factors and disease, they do not fully explain all cardiovascular events. To enhance our understanding and help with the management of CVD, investigations that involve the measurement of traditional as well as novel risk factors may be necessary. Public health strategies that aim to reduce the prevalence of obesity and overweight encourage youth to increase their physical activity levels as a means of protecting against poor cardiometabolic profiles. Interventions that increase physical activity levels and improve cardiorespiratory fitness cause a reduction in certain CVD risk factors but the lack of agreement between findings makes it impossible to give precise recommendations that will ensure CVD risk reduction. Yet it is important that research continues in order to establish the most appropriate means of improving the health and well-being of those at most risk of future CVD.
}

\section{Introduction}

Cardiovascular disease (CVD) has been the leading cause of death in developed countries since the early 1900s. ${ }^{1}$ While the risk of cardiovascular mortality was once relatively low in youth, accumulating evidence suggests that CVD has its origins in childhood. ${ }^{2,3}$ Numerous risk factors have been linked to the development of CVD and include; poor dietary habits, overweight and obesity, hypertension, insulin resistance or diabetes mellitus, alcohol consumption, smoking, poor physical activity levels and dyslipidemia. ${ }^{4}$ of relevance, many of these risk factors are related and reversible through a healthy diet and increased physical activity. Given that childhood obesity increases the risk of becoming obese in adulthood, and that obesity at any age is associated with numerous co-morbidities such as, type 2 diabetes, CVD risk, hypertension, asthma, depression, certain cancers, and sleep apnoea, ${ }^{1}$ reversing current trends in obesity prevalence seems vital.

More than 100 emerging risk factors have been suggested as potential markers for improving CVD risk classification. Several of these novel biomarkers have generated significant interest within the scientific community since they may provide additional means of improving CVD risk assessment. Some of these more widely investigated biomarkers include C-reactive protein (CRP), adiponectin, homocysteine, plasminogen activator inhibitor-1 (PAI1), interleukin-6 (IL-6) and fibrinogen (Fg). Much of the evidence linking these markers to CVD nonetheless has been derived from adult populations. Despite the extremely low risk of early mortality in youth, early and continued exposure to an unhealthy CVD profile may accentuate the risk of early mortality.

In order for public health strategies to be effective within youth populations, it may be necessary to evaluate both traditional and novel CVD risk factors to determine the most effective means of reducing unhealthy metabolic profiles. Thus, this review will provide an overview of the current literature concerning six of the widely investigated novel metabolic CVD risk factors while examining their associations with physical activity, obesity and measures of physical fitness.

\section{Adiponectin}

Adiponectin is an adipose specific hormone produced and secreted by adipose tissue which circulates in the blood in three forms (low, medium, and high), with the high-molecular-weight form appearing to be more pathogenic. ${ }^{5}$ Unlike other adipocytiknes (cell signalling proteins released by adipokines) such as interleukin-6 (IL-6) and plasminogen activator inhibitor-1 (PAI-1), adiponectin levels are lower in obese and overweight individuals. ${ }^{6}$ In adults, low 
levels of adiponectin have been known to predict outcomes such as CVD and type 2 diabetes ${ }^{7}$ and if found in childhood, may exacerbate the prevalence of these disorders when adulthood is reached. Obese children as young as 6-9 yrs have been known to have decreased adiponectin levels, in comparison to non obese children, with negative associations apparent for measures of BMI and IR ( both fasting insulin levels and HOMA). ${ }^{6}$ Puberty seems to have an effect on adiponectin levels with some studies demonstrating significant differences in adiponectin levels between and within genders during adolescence. $^{8}$

Data from the Québec Child and Adolescent Health and Social Survey which involved 1632 French Canadian youth aged 9, 13, and 16 yr demonstrated that for all age groups, boys displayed significantly lower concentrations of adiponectin in comparison to girls. ${ }^{8}$ Further analysis found that mean adiponectin levels were significantly lower at age 16 compared to 9 yrs for boys $(27.7 \%, \mathrm{P}<0.0001)$ and for girls $(13.3 \%, \mathrm{P}<0.0001)$ with changes in BMI the attributed cause. Similar findings were also seen in European children and adolescents with adiponectin levels being lower in lean male adolescents than girls of the same age. ${ }^{9}$ Interestingly the authors also measured testosterone levels and found that the significant difference noted between boys was inversely related to testosterone $(r=-$ $0.42 ; \mathrm{P}<0.0001)$ levels. It seems that differing testosterone levels apparent between genders during adolescence may explain apparent gender differences during this period.

Improving weight status seems important for healthy adiponectin levels in youth. Whether increasing physical activity levels and improving measures of physical fitness has the same beneficial effects are unclear. Though some have observed a positive effect of increased physical activity on adiponectin levels, ${ }^{10,11}$ others have not. ${ }^{12}$ In the study by Kim and colleagues, ${ }^{11}$ obese adolescents were involved in a 6 -week jump rope intervention. While the participants experienced significant improvements in adiponectin levels postintervention, significant improvements in body fat \% (\%BF) were experienced also. Similarly reductions in $\% \mathrm{BF}$ were also noted in the participants who experienced significant increases in adiponectin in the study by Balagapol and colleagues ${ }^{10}$ and seem to imply that a reduction in \%BF may be necessary for the effects of exercise upon adiponectin levels to become apparent in adolescents.

\section{Interleukin-6}

IL-6 is considered a pro-inflammatory and immunoregulating cytokine produced from adipose tissue. ${ }^{13}$ Resting levels of IL- 6 are known to be elevated in obese adults and are positively related to adiposity, particularly central body fat, while correlating with IR. ${ }^{14}$ Unsurprisingly, IL-6 has been proposed as playing a key role in the relationship between adiposity, inflammation, and CVD. Though one would expect elevated levels of IL-6 to be present in obese youth, findings have been somewhat contradictory. In a recent study involving European obese and overweight children (10 yrs), being overweight or obese was significantly associated with elevated levels of IL-6, the extent of which was exacerbated with increasing weight status. ${ }^{15}$ Similar findings were also seen in older participants (15-16 yrs) with obese adolescents demonstrating positive associations between IL-6 and measures of adiposity (body weight, BMI, and body fat mass) and IR (HOMA). ${ }^{16}$ Interestingly within this study, the obese participants also demonstrated similar positive associations with other pro-inflammatory biomarkers (C-reactive protein and fibrinogen).

Similarly, significant differences in the levels of IL- 6 were seen in severely obese children and adolescents from Austria $(12 \pm 4 \mathrm{yrs})$ when compared to their lean counterparts. Collectively, these studies seem to indicate the presence of an inflammatory state early in the clinical course of obesity. ${ }^{17}$ Nevertheless, others studies have not confirmed these assertions. ${ }^{13,18,19}$ In the study by Caballero et al. significant differences between obese and lean participants (10-18 yrs), of Hispanic (Latino) origin, were seen in concentrations of C-reactive protein, PAI-1 and adiponectin though no differences were noted for IL-6. Comparable findings were also seen in younger, pre-pubertal children. ${ }^{19}$ Here the authors demonstrated significant differences in C-reactive protein, insulin, PAI-1 levels and IR (HOMA) between obese and non-obese individuals though no differences were noted in IL-6 levels. In a recent study some have even suggested that IL-6 levels may be influenced by age and gender. ${ }^{13}$ In this longitudinal study conducted over 7 years, IL-6 levels were measured at 8 and 15 yrs in both healthy and overweight children. Though no significant differences were noted between the two groups at the first measurement, the authors did note that significant differences existed with regards to IR (HOMA). During adolescence significant differences were noted in females with the overweight participants having significantly higher IL-6 levels than their normal weight counterparts. Interestingly, no significant differences were noted between the boys at either measurement. ${ }^{18,19}$

Based on the contradictory findings of these studies it is difficult to establish the associations between IL- 6 and adiposity in youth populations. Though it is acknowledged that obese adults seem to present with elevated IL-6 levels in comparison to their leaner counterparts, making such an assertion in youth populations is difficult at this stage. Limited studies have focused on the associations between adiposity and IL-6 in healthy and overweight youth populations and until such case, further work is needed to establish this relationship. Nevertheless, as IL-6 is produced and secreted from adipose tissue it is sensible to expect that IL-6 levels should be raised in obese children and adolescents. It may be that the expression of elevated IL-6 levels in plasma is proceeded by other factors involved in the disease process as found by Tam and colleagues who noted the prevalence of IR in obese youth at 8 yrs despite comparable levels of IL- 6 in lean individuals with no prevalence of IR. ${ }^{13}$

Though studies reporting on the effects of physical activity and physical fitness on IL-6 levels in youth are sparse, some evidence does exists that supports the protective effects of both. In a group of 640 pre-adolescents ( $49 \%$ boys), Platat and others demonstrated that subjective measures of physical activity was independently and negatively associated with IL-6 after adjustment for sex, sexual maturity and socio-economic status. ${ }^{20}$ Though beneficial relationships were also noted between physical activity and C-reactive protein, adiponectin and leptin, it was interesting to note that only IR (HOMA) and IL-6 were the only significant associations after controlling for adiposity (body fat mass) and fat localization (waist-hipratio). However in two recent studies, objectively measured physical activity and cardiorespiratory fitness was not shown to be associated with IL-6 levels in either 13-17 yrs individuals ${ }^{21}$ or in 9 yr olds. ${ }^{22}$ This was found despite measures of adiposity and low cardiorespiratory fitness being shown to predict the clustering of CVD risk (composite score produced from measures of systolic BP, triglyceride, IR (HOMA), and cholesterol to HDL ratio) ${ }^{22}$ and elevated C-reactive protein levels and IR (HOMA). ${ }^{21}$ Though Andersen and colleagues suggest that the reason for the lack of association noted in their study between IL-6 and CVD risk may relate to the young age of participants whereby not enough time may have lapsed for this association to develop, this was not supported in the study by Martinez-Gomes and colleagues. Clearly, more longitudinal research seems warranted to determine the associations and effects of IL-6 levels upon indices of CVD risk during youth and subsequent adulthood. 


\section{Homocysteine}

Homocysteine is a sulphur-containing amino acid derived from methionine, with elevated levels known to be linked to endothelial damage, platelet activation and altered thrombus formation. ${ }^{23}$ At present there is no international consensus regarding a cut-off point for hyperhomocysteinaemia in youth populations though the American Heart Association have proposed a normal range for fasting homocysteine of between $5-15 \mu \mathrm{mol} / \mathrm{L}$ in adults. ${ }^{24}$ Though there is a dearth of evidence relating to youth compared to adults, the available evidence does appear to concur with these ranges..$^{25-27}$ In a study by Kerr and colleagues, ${ }^{26}$ the authors used data from the National Dietary and Nutritional Survey of 2127 youths aged between 4 to 18 years to produce age-specific reference values for homocysteine. From their findings, homocysteine concentrations were found to progressively increase with age with median values of $5.6,6.3$, and $7.9 \mu \mathrm{mol} / \mathrm{L}$ noted in youth aged 4 to 10 years, 11 tol4 years, and 15 to 18 years, respectively. Ranges for total homocysteine have also been presented by Reddy ${ }^{27}$ for apparently healthy youth aged between 9 and 14 years of $8.3 \pm 3.7 \mu \mathrm{mol} / \mathrm{L}$ in boys and $8.3 \pm 3.3$ $\mu \mathrm{mol} / \mathrm{L}$ in girls though the differences in ages between the two cohorts make comparison difficult. It does appear nonetheless that specific gender differences do exist in youth with boys typically presenting with significantly higher levels of homocysteine than girls. ${ }^{26,28,29}$

The age at which specific gender differences in homocysteine levels become apparent is unclear. Some have noted specific gender differences from the age of 15 years, ${ }^{26,29}$ while others have reported significant gender differences in homocysteine levels at a younger age. ${ }^{25,28}$ One possible explanation for the apparent discrepancies between studies may relate to the levels of gender specific hormones evident from the onset of puberty. For instance, the rise in oestrogen levels in females at the onset of puberty is known to be protective against the rise in homocysteine levels, ${ }^{30}$ whereas the rise in testosterone levels in males has been postulated as a possible explanation for their elevated homocysteine levels. ${ }^{31}$ Whether these apparent gender differences pose a significant risk to future health is unclear given that the role of homocysteine has yet to be conclusively confirmed as a risk factor for future CVD. ${ }^{26}$ Nonetheless, hyperhomocysteinaemia, which can be defined as an elevated concentration of fasting plasma total homocysteine, has been associated with an increase in the possibility of suffering from CVD related disorders later in life. ${ }^{25,32}$ Importantly though, homocysteine levels are known to be modifiable.

Lifestyle factors such as poor physical activity levels, smoking status, excessive alcohol consumption and obesity have all been associated with elevated levels of homocysteine in adults. ${ }^{33,34}$ Previous investigations have also demonstrated that homocysteine concentrations are associated with indices of childhood obesity, ${ }^{35,36}$ but whether the associations between homocysteine concentrations and physical activity and cardiorespiratory fitness as in adults, is unclear. To date, limited studies are available that have examined the possible associations between physical activity, physical fitness and homocysteine levels in youth. However, in a study involving 156 Spanish adolescents (76 boys and 80 girls), Ruiz and colleagues did examine the associations between .cardiorespiratory fitness as seen in adults is evident in youth, is unclear. From their findings, the authors rather interestingly noted a significant inverse association between homocysteine and cardiorespiratory fitness in girls though no associations were apparent in boys. ${ }^{37}$ These findings have also been noted in a previous investigation involving adults, ${ }^{38}$ and seem to suggest that sex specific hormones may play a role in mediating the homocysteine-cardiorespiratory fitness relationship. ${ }^{37}$
To the best of our knowledge, no studies have examined the independent effects of a physical activity intervention upon homocysteine levels in youth. Gallistl and colleagues ${ }^{36}$ did however demonstrate a positive effect on the homocysteine levels in obese children (aged 10-14 years) after only 3 weeks of a combined physical activity and dietary intervention. Nonetheless, the lack of information provided concerning the physical activity performed means that it is difficult to elucidate the effects of the activity upon homocysteine levels, particularly when measures of adiposity significantly decreased post-intervention also. It is clear that further investigations are required to determine the extent increasing physical activity and or cardiorespiratory fitness has upon homocysteine levels in youth. Furthermore, longitudinal tracking studies are also required to confirm whether a relationship between youth and adult levels of homocysteine is apparent and indeed whether these levels in youth have any impact upon CVD risk in adulthood.

\section{Plasminogen activator inhibitor-1}

PAI-1 is a globular glycoprotein with a molecular weight of 50,000; it is comprised of 379 amino acids in a single chain whose structure designates it as a member of the superfamily of serine protease inhibitors (serpins). ${ }^{23}$ Previous investigations have established that higher circulating PAI-1 levels are associated with increased risk of CVD and are associated with with obesity, hyperinsulinemia and type 2 diabetes. ${ }^{24}$ The synthesis and production of PAI-1 mainly occurs through endothelial cells though other types of tissue produce PAI-1 such as hepatocytes and adipocytes. The underlying mechanism that results in elevated PAI-1 levels is a complex process and at present is not fully understood. A detailed discussion of this is out with the scope of this paper and the reader is directed towards two detailed review articles for a more comprehensive discussion on this matter. ${ }^{25,26}$ of particular interest though is the link between elevated PAI-1 levels and obesity. Raised PAI-1 levels in adults consistently appear in obese individuals and are known to increase the susceptibility of atherothrombotic events in such individuals. In blood, fibrinolysis breaks down fibrin or blood clots and is essential to prevent adverse thrombus formation. PAI-1 is known to be a potent inhibitor of fibrinolysis with elevated levels consistently found in obese individuals and is known to predict the occurrence of myocardial infarction. ${ }^{24}$ The metabolic syndrome is a cluster of metabolic abnormalities that can co-occur in an individual. Such abnormalities include obesity (visceral), hyperinsulinemia, dyslipidemia, elevated triglyceride levels, impaired glucose tolerance, hypertension and low HDL-C levels which are all well established risk factors for CVD. ${ }^{27}$ Evidence now suggests that elevated PAI-1 levels are linked to the metabolic syndrome as well as type 2 diabetes. ${ }^{25}$ However, PAI-1 is not associated with all components of the metabolic syndrome. Though studies have demonstrated a positive association between PAI-1 levels and measures of adiposity (BMI and WC), IR and hyperglycemia, ${ }^{28}$ it is not associated with dyslipidemia. ${ }^{25}$ Previous studies have also demonstrated a threefold increase in PAI1 levels in obese diabetic subjects when compared to lean counterparts ${ }^{29}$ and predictably, measures of adiposity, and in particular central adiposity, are seen as one of the main factors associated with impaired fibrinolysis and elevated PAI-1 levels.

Given the associations between the metabolic syndrome and elevated levels of PAI-1 it is prudent to recommend a treatment of lifestyle modifications in order to address both. Thus, the cornerstone of such an approach lies in modifying dietary habits and increasing physical activity levels. Previous investigations in adults have shown that dietary interventions can lead to significant reductions in PAI-1 levels. In the study by Mavri and others the authors demonstrated that after only a 3 -month weight reduction programme 
consisting of a hypocaloric diet, obese adults experienced significant reductions (-74\%) in PAI-1 levels. Interestingly, the changes in PAI1 activity was also associated with reductions in insulin levels and measures of adiposity (BMI and \%BF). ${ }^{30}$

In a similar study of induced energy intake restriction lasting for 11 weeks, significant improvements in PAI-1 levels (-50\%) were seen in obese elderly women after reductions in body weight and fat mass. ${ }^{31}$ Studies investigating the effects of increased physical activity on PAI-1 levels however are scarce. Though previous investigations have demonstrated substantial improvements in PAI-1 levels with increased physical activity, both of these studies have also involved a dietary modification programme too. ${ }^{32,33}$ The independent effects of increased physical activity on PAI-1 levels are thus impossible to elucidate. Nevertheless, it is interesting to note that in both of these studies, reductions in PAI-1 levels were accompanied by significant weight loss and a reduction in plasma insulin levels though this did not reach significance in the latter study.

In one of the few investigations that has looked at the independent effects of increased physical activity upon PAI-1 in adults aged between 50 and $70 \mathrm{yrs}$, the authors demonstrated no decrease in levels after 10 consecutive days of moderate intensity exercise training. ${ }^{34}$ Further, the authors also observed no significant reductions in body weight after the intervention period. Closer inspection of the results revealed that those individuals with higher body fatness did however experience significant reductions in insulin levels post intervention, unlike their leaner counterparts. Though speculative, it appears that the maintenance of body weight during the intervention period may explain the lack of positive effect upon PAI-1 activity. Within youth populations, evidence also supports the benefits of behaviour lifestyle modifications upon PAI-1 levels. In a study involving 60 obese pre-adolescents, ${ }^{35}$ significant reductions in elevated PAI-1 levels were seen after only 3 weeks of a programme, which consisted of a low-caloric diet and increased physical activity. Once more reductions in PAI-1 levels were associated with a reduction in measures of BMI whereas those not experiencing a decrease in adiposity saw no decrease in PAI-1 activity.

In a more recent study, the effects of a 6 -month lifestyle intervention upon on a number of CVD risk factors, including PAI-1 levels, in eighty five obese adolescents from Mexico was examined. ${ }^{36}$ Analysis of baseline results between the obese $(n=86)$ and lean $(n=36)$ groups revealed significant differences in triglycerides, glucose, insulin, PAI-1 and fibrinogen levels as one would expect. Post intervention, the obese adolescents who experienced significant reductions in BMI also experienced significant reductions in fasting insulin and PAI-1 levels. While this is an exciting finding, the nature of the study and of those previously discussed highlights the limitations of our understanding of the effects of lifestyle behaviours upon PAI-1 levels.

For instance in the study by Huang and colleagues there was no measures taken on participants to ensure whether they complied with the dietary and physical activity recommendations. The authors recommended that participants perform brisk walking for 30 min daily during the first 2 weeks with the duration of activity increasing to $1 \mathrm{~h}$ in the $3^{\text {rd }}$ week and thereafter until the completion of the intervention. Participants were also encouraged to undertake a variety of activities such as cycling, jogging and swimming in addition to the prescribed walking requirements. However it does not seem that this was recorded by the participants or indeed by the authors. So whether the effects of improved dietary habits and/or increased physical activity resulted in the improvements noted is unclear. The same concerns can be raised at the previous investigations mentioned within this section. As improvements in weight status and adiposity are associated with improvements in fasting glucose and insulin levels, it may be that the changes evident in these metabolic indices are responsible for the improvements noted in fibrinolytic activity. Given the dearth of studies available involving youth participants, it is unclear whether the relationship between a reduction in measures of adiposity and decreased PAI-1 levels is causal or indirect. Further studies seems warranted to understand the independent effects of improvements in measures of adiposity and increased physical activity upon PAI-1 levels before recommendations can be made. Another important limitation of this topic is the use of obese children and adolescents. While one can argue that these individuals are in most need of intervention, the limited data available concerning PAI-1 activity in youth limits the generalizability of findings to non-obese populations. Further work needs to consider whether improvements in PAI-1 levels are needed in non-obese cohorts and whether improvements are noted in the absence of a reduction in measures of adiposity.

\section{Fibrinogen}

Fibrinogen is a soluble glycoprotein found in plasma, which is predominantly synthesised in the liver. Fibrinogen is the precursor of fibrin and is an essential component of the blood coagulation system and is known to play a vital role in a number of pathophysiological processes within the body including inflammation, atherogenesis and thrombogenesis. ${ }^{37}$ Several studies have reported that plasma fibrinogen levels are an independent risk factor for $\mathrm{CVD},{ }^{38,39}$ though the exact mechanism whereby elevated fibrinogen levels leads to atherothrombosis is unclear.

Though fibrinogen is the precursor of fibrin, it is also an acute phase protein whose release from the liver is mediated in response to cytokines released in response to inflammation, infection and/or tissue damage. For instance, it is known that increased IL-6 production leads to an increase in fibrinogen synthesis whereas increased levels of circulating PAI-1 can lead to hypofibrinolysis, a state in which fibrinolysis is inhibited. ${ }^{5}$ Thus in addition to its vital role in the final phase of the blood coagulation cascade, fibrinogen is also known to promote platelet aggregation, smooth muscle cell migration and proliferation while also regulating whole blood and plasma viscosity. ${ }^{5}$

No specific cut-off values are available for youth populations though in adults, normal fibrinogen values range are said to range from 150 to $350 \mathrm{mg} / \mathrm{dL}{ }^{40}$ It should be noted though that fibrinogen levels are influenced by numerous factors such as age, gender, socioeconomic status, alcohol consumption, physical activity behaviour, cardiorespiratory fitness and measures of adiposity, ${ }^{37}$ which need to be considered when identifying at risk individuals. Previous investigations in youth populations have revealed that elevated fibrinogen concentrations are independently associated with measures of adiposity, cardiorespiratory fitness and physical activity. For instance, findings from the Bogalusa Heart study have shown the ponderal index (weight/ height ${ }^{3}$ ) and HDL-C to be independently associated with fibrinogen in a cohort of 3047 children and adolescents of mixed race and gender. ${ }^{41}$ Though fibrinogen levels measured in this cohort (5 to 17 yrs) revealed no significant race and sex differences, it was interesting to note that levels did increase significantly with age and sexual maturation in African-American females.

Similarly, in a smaller cohort of obese children $(n=41)$ Ferguson and colleagues noted significant differences in fibrinogen levels between gender and race. ${ }^{42}$ Here boys were seen to have significantly higher fibrinogen levels than girls whereas African-Americans had significantly greater levels than Caucasians. Further analysis revealed that gender and $\% \mathrm{BF}$ explained significant amounts of the variance in fibrinogen levels which led the authors to conclude the debilitating role adiposity plays in youth blood haemostasis.

Measures of adiposity (BMI, WC, WHR and \%BF) have all been 
shown to be positively associated with levels of fibrinogen in adult populations regardless of gender. ${ }^{37,43}$

The similar findings in youth populations seem to confirm adiposity as a key contributor to elevated fibrinogen levels and implicate the significance of modifying lifestyles as a key area for intervention. Though one would expect a positive association upon fibrinogen levels with increased physical activity and cardiorespiratory fitness in youth, cross-sectional evidence has revealed contradictory findings in youth populations. ${ }^{44-48}$

For instance, the positive associations seen between subjective measures of physical activity and fibrinogen in children and adolescents of differing gender and ethnicity have to be viewed with caution as both failed to consider the potential effects of co-founders in their analysis. ${ }^{45,47}$ When objective measures of physical activity were captured in children however, no association was seen between physical activity and fibrinogen levels after controlling for potential co-founders (sex, age and pubertal development). ${ }^{44}$ Similarly with regards to cardiorespiratory fitness, the independent associations noted with physical activity in a range of individuals aged between 4 and $25 \mathrm{yrs}^{46}$ was not confirmed in other studies involving obese adolescent boys and girls ${ }^{48}$ or in pre-pubertal boys and girls.44 Differences in methodologies and sample participants may explain the conflicting findings from these studies but further work is needed to substantiate whether an association exists between physical activity and measures of physical fitness and fibrinogen concentrations in youth populations.

\section{C-reactive protein}

CRP is a circulating acute-phase reactant whose production increases significantly in response to trauma or inflammation. Synthesized primarily in the liver, its release is principally stimulated by IL- 6 and TNF- $\alpha$ production which reveals a direct link between adipocytes and CRP. ${ }^{5}$ It is well established that inflammation is central to CVD risk with elevated levels of inflammatory cytokines known to be associated with atherosclerosis, with CRP being one of the most sensitive indicators. ${ }^{49}$ Some have suggested that traditional CVD risk factors only explain around $50 \%$ of cardiovascular morbidity and mortality, ${ }^{50}$ thus, the importance and utility of further predictors such as CRP being used as an additional means of detecting unfavourable lifestyle behaviours is now accepted.

Inflammation resulting from injury, infection or irritation can be deemed as either acute or chronic. Acute inflammation refers to the body's initial response to harmful stimuli caused either through infection of trauma whereas chronic inflammation caused through obesity, arthritis and atherosclerosis may last days or several years resulting in the influx of inflammatory cells at the localized tissue. ${ }^{49}$ In both adults and youth populations, chronic inflammation is a common characteristic of being overweight and obese. Adipose tissue is known to release a number of inflammatory biomarkers into the blood stream, notably IL-6, which in turn can stimulate the release of CRP from the liver into the blood stream. ${ }^{5}$ Given the association between inflammation and the pathogenesis of disease, the study of $\mathrm{CRP}$ as a predictor of future CVD events has received much attention in recent years.

However, much of our understanding of CRP and its role in the progression of atherosclerosis has come from the study of adults. Nonetheless, the association between obesity and CRP levels is now firmly established in both children and adolescents. In a cross sectional study involving 699 British children (10-11 yrs), Cook and colleagues ${ }^{51}$ were the first to demonstrate the association between obesity and inflammatory biomarkers. Findings revealed that CRP levels were $47 \%$ higher in girls than in boys and when compared by age groups, levels were seen to rise by $15 \%$. Importantly though, CRP lev- els were seen to be $270 \%$ higher in individuals who were in the top quintile rather than the bottom quintile of the Ponderal index. Though CRP was seen to be associated with several CVD risk factors measured in this cohort, only the associations for fibrinogen, HDL-C and systolic blood pressure held true after adjustment for adiposity. Interestingly, further analysis revealed that adiposity was significantly associated with all CVD risk factors measured (fasting glucose, fibrinogen, triglyceride, total cholesterol, HDL-C, LDL-C and insulin levels as well as systolic and diastolic blood pressure), even after adjustment for age, gender, socio-economic status, and CRP levels. These findings seem to imply that adiposity may affect CVD risk factors through other pathways than those for CRP.

Similar findings have also been reported in older cohorts (8-16 yrs) with overweight boys and girls presenting with CRP levels of 3.74 and 3.17 times greater than their normal weight counterparts. ${ }^{52}$ Longitudinal prospective studies have also demonstrated that CRP levels track significantly from childhood into adulthood i.e. ages 3-18 to ages $24-39$ yrs $^{53}$ and from $16-25$ yrs with Morrison and colleagues noting unhealthy changes to BMI, WC and total cholesterol as being the major determinants of CRP levels in adulthood. ${ }^{54}$ Though findings from the Young Finns study revealed that childhood CRP levels was not associated with adult carotid artery intimal medial thickness (cIMT), ${ }^{53}$ others have found significant associations between CRP levels and measures of atherosclerosis [cIMT and Brachial artery flow-mediated dilatation (FMD)] in healthy children $(10.5 \pm 1.1$ yrs). ${ }^{55}$

Collectively, these findings support the predictive validity of CRP measurement in identifying those at risk of current and/or future CVD. With the established association between CRP and measures of adiposity it not surprising that some have suggested that increasing physical activity and cardiorespiratory fitness levels may prove useful in reducing CRP levels in youth populations. However, current evidence is scant and rather contradictory with regards to their mediating role upon youth CRP levels.

Though some have shown significant associations between physical activity and CRP levels ${ }^{47,51}$ others have not. ${ }^{44}$ Nevertheless, the evidence should be viewed with caution given the limitations of subjective self-report measures and the failure to consider potential cofounders such as measures of adiposity in some of these studies. When physical activity has been measured using objective methods (accelerometers) though, no associations were apparent with CRP in a cohort of Swedish pre-pubertal children. ${ }^{44}$ The authors did however find a negative association between CRP and cardiorespiratory fitness and a positive association with body fat, which suggests that physical activity may have a protective role on low-grade inflammation through reductions in body fat and enhancement of cardiorespiratory fitness. Similar contradictory evidence is apparent when examining the associations between cardiorespiratory fitness and CRP levels in youth.

Though authors have demonstrated significant inverse associations between cardiorespiratory fitness and CRP levels in both prepubertal and adolescents boys and girls ${ }^{22,56}$ others have not. ${ }^{57,58}$ One possible explanation for the contradictory results may relate to the methods used to measure cardiorespiratory fitness. In the former studies cardiorespiratory fitness was measured on a treadmill through continuous and progressive protocols whereas in the latter studies the 20-m multistage fitness test (20-m MSFT) was used as an estimate. Though laboratory measures may have produced a more accurate measurement, the 20 -m MSFT is accepted as a valid and reliable test of cardiorespiratory fitness in young people and since students are familiar with the test protocol, it is presumed that the levels achieved are reliable estimates. 


\section{Conclusions}

CVD continues to be the leading cause of death in the Western world. As the prevalence of CVD risk factors seem to becoming more evident in childhood, it is vital that pre-emptive interventions are implemented early in one's life before associated cardiovascular complications are established. As physical activity can influence obesity directly, and given the associations between obesity and CVD risk, it is important that we endeavor to disentangle this causal relationship. Individuals that are physically active and are of a healthy weight and high cardiorespiratory fitness decrease the likelihood of early mortality and cardiovascular morbidity. Nevertheless, it is clear from the literature discussed that our understanding of the significance and associations between pre-clinical markers of CVD and obesity, physical activity and physical fitness in youth is poorly understood. Findings are often contradictory which makes it difficult to assert conclusively the best means of reversing potentially unfavourable behaviours and cardiovascular and metabolic profiles. Nonetheless, the relative importance of identifying specific risk factors in the prevention of CVD related disorders later in life requires continued perseverance.

Improved understanding of the effects of physical activity, physical fitness and obesity upon CVD risk factors is vital. The school environment, and in particular physical education, affords an ideal setting to practice health-promoting behaviours and is widely recognized as an important setting for intervention. ${ }^{59}$ Moreover, the school setting provides a vehicle whereby interventions can reach a large number of individuals from assorted socio-economic surroundings; avoid stigmatisation while affording an environment for youth to partake in healthy behaviours. It is important therefore that research continues to examine the best means of reversing poor metabolic profiles in youth so that appropriate strategies can be implemented that will the health and well-being of all individuals.

Correspondence: Dr. Duncan S. Buchan, Health and Exercise Sciences, School of Science, University of the West of Scotland, Hamilton, ML3 0JB, Scotland, UK. Tel. +44.1698.283100 Email: duncan.buchan@uws.ac.uk Key words: cardiovascular disease, youth, physical fitness, physical activity and obesity.

Conflict of interest: the authors declare no conflicts of interest.

Received for publication: 21 November 2011.

Accepted for publication: 27 January 2012.

CC Copyright D.S. Buchan et al., 2012

Licensee PAGEPress, Italy

Journal of Public Health Research 2012; 1:e11

doi:10.4081/jphr.2012.e11

This work is licensed under a Creative Commons Attribution NonCommercial

3.0 License (CC BY-NC 3.0).

\section{References}

1. World Health Organization. The challenge of obesity in the WHO European Region and the strategies for response. 2007, World Health Organisation Copenhagen, Denmark. Accessed on: 21 July 2011. Available from: http://www.euro.who.int/data/assets/ pdf_file/0010/74746/E90711.pdf

2. Raitakari OT, Juonala M, Kahonen M, et al. Cardiovascular risk factors in childhood and carotid artery intima-media thickness in adulthood: the cardiovascular risk in young Finns study. JAMA 2003;290:2277-83.

3. Andersen LB, Harro M, Sardinha LB, et al. Physical activity and clustered cardiovascular risk in children: a cross-sectional study (The European Youth Heart Study). Lancet 2006;368:299-304.

4. Ortega FB, Artero EG, Ruiz JR, et al. Physical fitness levels among European adolescents: the HELENA study. Br J Sports Med 2011;45:20-9.

5. Balagopal PB, de Ferranti SD, Cook S, et al. Nontraditional risk factors and biomarkers for cardiovascular disease: mechanistic, research, and clinical considerations for youth: a scientific statement from the American Heart Association. Circulation 2011;123:2749-69.

6. Valle M, Martos R, Gascon F, et al. Low-grade systemic inflammation, hypoadiponectinemia and a high concentration of leptin are present in very young obese children, and correlate with metabolic syndrome. Diabetes Metab 2005;31:55-6.

7. Steinberger J, Daniels SR, Eckel RH, et al. Progress and challenges in metabolic syndrome in children and adolescents: a scientific statement from the American Heart Association atherosclerosis, hypertension, and obesity in the Young Committee of the Council on cardiovascular disease in the young; Council on cardiovascular nursing; and Council on nutrition, physical activity, and metabolism. Circulation 2009;119:628-47.

8. Punthakee Z, Delvin EE, O'Loughlin J, et al. Adiponectin, adiposity, and insulin resistance in children and adolescents. J Clin Endocrinol Metab 2006;91:2119-25.

9. Bottner A, Kratzsch J, Muller G, et al. Gender differences of adiponectin levels develop during the progression of puberty and are related to serum androgen levels. J Clin Endocrinol Metab 2004;89:4053-61.

10. Balagopal P, George D, Yarandi H, et al. Reversal of obesity-related hypoadiponectinemia by lifestyle intervention: a controlled, randomized study in obese adolescents. J Clin Endocrinol Metab 2005;90:6192-7.

11. Kim ES, Im JA, Kim KC, et al. Improved insulin sensitivity and adiponectin level after exercise training in obese Korean youth. Obesity (Silver Spring) 2007;15:3023-30.

12. Metcalf BS, Jeffery AN, Hosking J, et al. Objectively measured physical activity and its association with adiponectin and other novel metabolic markers: a longitudinal study in children (EarlyBird 38). Diabetes Care 2009;32:468-73.

13. Tam CS, Garnett SP, Cowell CT, et al. IL-6, IL-8 and IL-10 levels in healthy weight and overweight children. Horm Res Paediatr 2010;73:128-34.

14. Liu S, Tinker L, Song Y, et al. A prospective study of inflammatory cytokines and diabetes mellitus in a multiethnic cohort of postmenopausal women. Arch Intern Med 2007;167:1676-85.

15. Nagel G, Rapp K, Wabitsch M, et al. Prevalence and cluster of cardiometabolic biomarkers in overweight and obese schoolchildren: results from a large survey in southwest Germany. Clin Chem 2008;54:317-25.

16. Balagopal P, George D, Patton N, et al. Lifestyle-only intervention attenuates the inflammatory state associated with obesity: a ran- 
domized controlled study in adolescents. J Pediatr 2005;146:342 8.

17. Kapiotis S, Holzer G, Schaller G, et al. A proinflammatory state is detectable in obese children and is accompanied by functional and morphological vascular changes. Arterioscler Thromb Vasc Biol 2006;26:2541-6.

18. Caballero AE, Bousquet-Santos K, Robles-Osorio L, et al. Overweight Latino children and adolescents have marked endothelial dysfunction and subclinical vascular inflammation in association with excess body fat and insulin resistance. Diabetes Care 2008;31:576-82.

19. Valle Jimenez M, Estepa RM, Camacho RM, et al. Endothelial dysfunction is related to insulin resistance and inflammatory biomarker levels in obese prepubertal children. Eur J Endocrinol 2007;156:497-502.

20. Platat C, Wagner A, Klumpp T, et al. Relationships of physical activity with metabolic syndrome features and low-grade inflammation in adolescents. Diabetologia 2006;49:2078-85.

21. Martinez-Gomez D, Eisenmann JC, Warnberg J, et al Associations of physical activity, cardiorespiratory fitness and fatness with low-grade inflammation in adolescents: the AFINOS Study. Int J Obes (Lond) 2010;34:1501-7.

22. Andersen LB, Muller K, Eiberg S, et al. Cytokines and clustered cardiovascular risk factors in children. Metabolism 2010;59:5616 .

23. Erickson LA, Ginsberg MH, Loskutoff DJ. Detection and partial characterization of an inhibitor of plasminogen activator in human platelets. J Clin Invest 1984;74:1465-7.

24. Smith A, Patterson C, Yarnell J, et al. Which hemostatic markers add to the predictive value of conventional risk factors for coronary heart disease and ischemic stroke? The Caerphilly Study. Circulation 2005;112:3080-7.

25. Alessi MC, Juhan-Vague I. PAI-1 and the metabolic syndrome: links, causes, and consequences. Arterioscler Thromb Vasc Biol 2006;26:2200-7.

26. Kerr MA, Livingstone B, Bates CJ, et al. Folate, related B vitamins, and homocysteine in childhood and adolescence: potential implications for disease risk in later life. Pediatrics 2009;123:627-35.

27. Reddy MN. Reference ranges for total homocysteine in children. Clin Chim Acta 1997;262:153-5.

28. Meigs JB, Barry MJ, Giovannucci E, et al. Incidence rates and risk factors for acute urinary retention: the health professionals followup study. J Urol 1999;162:376-82.

29. McGill JB, Schneider DJ, Arfken CL, et al. Factors responsible for impaired fibrinolysis in obese subjects and NIDDM patients. Diabetes 1994;43:104-9.

30. Mavri A, Stegnar M, Krebs M, et al. Impact of adipose tissue on plasma plasminogen activator inhibitor-1 in dieting obese women. Arterioscler Thromb Vasc Biol 1999;19:1582-7.

31. Calles-Escandon J, Ballor D, Harvey-Berino J, et al. Amelioration of the inhibition of fibrinolysis in elderly, obese subjects by moderate energy intake restriction. Am J Clin Nutr 1996;64:7-11.

32. Lindahl B, Nilsson TK, Asplund K, Hallmans G. Intense nonpharmacological intervention in subjects with multiple cardiovascular risk factors: decreased fasting insulin levels but only a minor effect on plasma plasminogen activator inhibitor activity. Metabolism 1998;47:384-90.

33. Lindahl B, Nilsson TK, Jansson JH, et al. Improved fibrinolysis by intense lifestyle intervention. A randomized trial in subjects with impaired glucose tolerance. J Intern Med 1999;246:105-12.

34. Bodary PF, Yasuda N, Watson DD, et al. Effects of short-term exercise training on plasminogen activator inhibitor (PAI-1). Med Sci Sports Exerc 2003;35:1853-8.
35. Sudi KM, Gallistl S, Trobinger M, et al. The influence of weight loss on fibrinolytic and metabolic parameters in obese children and adolescents. J Pediatr Endocrinol Metab 2001;14:85-94.

36. Gallistl S, Sudi KM, Borkenstein M, et al. Determinants of haemostatic risk factors for coronary heart disease in obese children and adolescents. Int $\mathrm{J}$ Obes Relat Metab Disord 2000;24:1459-64.

37. Ruiz RR, Sola R, Gonzalez-Gross M, et al. Cardiovascular fitness is negatively associated with homocysteine levels in female adolescents. Arch Pediatr Adolesc Med 2007;161:166-71.

38. Danesh J, Lewington S, Thompson SG, et al. Plasma fibrinogen level and the risk of major cardiovascular diseases and nonvascular mortality: an individual participant meta-analysis. JAMA 2005;294:1799-809.

39. Danesh J, Collins R, Appleby P, Peto R. Association of fibrinogen, C-reactive protein, albumin, or leukocyte count with coronary heart disease: meta-analyses of prospective studies. JAMA 1998;279:1477-82.

40. El-Sayed MS. Fibrinogen levels and exercise. Is there a relationship? Sports Med 1996;21:402-8.

41. Bao W, Srinivasan SR, Berenson GS. Plasma fibrinogen and its correlates in children from a biracial community: the Bogalusa Heart Study. Pediatr Res 1993;33(4 Pt 1):323-6.

42. Ferguson MA, Gutin B, Owens S, et al. Fat distribution and hemostatic measures in obese children. Am J Clin Nutr 1998;67:1136 40.

43. Krobot K, Hense HW, Cremer P, et al. Determinants of plasma fibrinogen: relation to body weight, waist-to-hip ratio, smoking, alcohol, age, and sex. Results from the second MONICA Augsburg survey 1989-1990. Arterioscler Thromb 1992;12:780-8.

44. Ruiz JR, Ortega FB, Warnberg J, Sjostrom M. Associations of lowgrade inflammation with physical activity, fitness and fatness in prepubertal children; the European Youth Heart Study. Int J Obes 2007;31:1545-51.

45. Zahavi I, Yaari S, Salman H, et al. Plasma fibrinogen in Israeli Moslem and Jewish school-children: distribution and relation to other cardiovascular risk factors. The Petah Tikva project. Isr J Med Sci 1996;32:1207-12.

46. Isasi CR, Starc TJ, Tracy RP, Deckelbaum R, Berglund L, Shea S. Inverse association of physical fitness with plasma fibrinogen level in children: the Columbia University BioMarkers Study. Am J Epidemiol. 2000 Aug 1;152(3):212-8.

47. Syrenicz A, Garanty-Bogacka B, Syrenicz M, et al. Low-grade systemic inflammation and the risk of type 2 diabetes in obese children and adolescents. Neuroendocrinol Lett 2006;27:453-8.

48. Gallistl S, Sudi KM, Borkenstein M, et al. Determinants of haemostatic risk factors for coronary heart disease in obese children and adolescents. Int $\mathrm{J}$ Obes Relat Metab Disord 2000;24:1459-64.

49. Tam CS, Clement K, Baur LA, Tordjman J. Obesity and low-grade inflammation: a paediatric perspective. Obes Rev 2010;11:11826.

50. Wei M, Mitchell BD, Haffner SM, Stern MP. Effects of cigarette smoking, diabetes, high cholesterol, and hypertension on allcause mortality and cardiovascular disease mortality in Mexican Americans. The San Antonio Heart Study. Am J Epidemiol 1996;144:1058-65.

51. Cook DG, Mendall MA, Whincup PH, et al. C-reactive protein concentration in children: relationship to adiposity and other cardiovascular risk factors. Atherosclerosis 2000;149:139-50.

52. Visser M, Bouter LM, McQuillan GM, et al. Low-grade systemic inflammation in overweight children. Pediatrics 2001;107:E13.

53. Juonala M, Viikari JS, Ronnemaa T, et al. Childhood C-reactive protein in predicting CRP and carotid intima-media thickness in 
adulthood: the cardiovascular risk in young Finns study. Arterioscler Thromb Vasc Biol 2006;26:1883-8.

54. Morrison JA, Glueck CJ, Daniels SR, et al. High-sensitivity C reactive protein: associations with cardiovascular risk factors and tracking in female adolescents and young adults. ISRN Pediatrics vol. 2011, Article ID 707206.

55. Jarvisalo MJ, Harmoinen A, Hakanen M, et al. Elevated serum Creactive protein levels and early arterial changes in healthy children. Arterioscler Thromb Vasc Biol 2002;22:1323-8.

56. Isasi CR, Deckelbaum RJ, Tracy RP, Set al. Physical fitness and C-reactive protein level in children and young adults: the Columbia University BioMarkers Study. Pediatrics 2003;111:3328.
57. Warnberg J, Nova E, Moreno LA, et al. Inflammatory proteins are related to total and abdominal adiposity in a healthy adolescent population: the AVENA Study. Am J Clin Nutr 2006;84:505-12.

58. Thomas NE, Cooper SM, Williams SP, Baker JS, Davies B. Relationship of fitness, fatness, and coronary-heart-disease risk factors in 12- to 13-year-olds. Pediatr Exerc Sci. 2007 Feb;19(1):93-101.

59. World Health Organization. Global Recommendations on Physical Activity for Health. 2010, World Health Organization, Geneva, Switzerland. Accessed on: 8 August 2011. Available from: http://whqlibdoc.who.int/publications/2010/9789241599979_eng. pdf 